\title{
RECENT DEVELOPMENTS IN MINIEMULSION POLYMERIZATION
}

\author{
DESARROLLOS RECIENTES EN POLIMERIZACION EN MINIEMULSIONES
}

I. Aizpurua1 , J.I. Amalvy², M.J. Barandiaran', J.C. de la Cal $^{1}$ and J.M. Asua ${ }^{1}$

\section{SUMMARY}

Some developments in miniemulsion polymerization aiming at taking advantage of its unique mechanisms minimizing the drawbacks of this technique are discussed. The discussion includes preparation of highly concentrated latexes, miniemulsion polymerization in continuous stirred tank reactors (CSTRs), and elimination of the low molecular weight hydrophobe.

\section{INTRODUCTION}

Miniemulsions are finely divided oil-in-water dispersions stabilized against coagulation by conventional emulsifiers (usually anionic emulsifiers) and containing a highly water-insoluble compound (hydrophobe) to minimize the Ostwald ripening effect, namely, the diffusion of oil phase from small to large droplets to minimize the interfacial free energy of the system. Monomer miniemulsions can be polymerized upon addition of either water-soluble or oil-soluble initiators leading to a dispersed polymer, similar to a latex produced by conventional emulsion polymerization [1-19]. Nevertheless, miniemulsion polymerization follows a mechanism different from emulsion polymerization. The main difference is that, in miniemulsion polymerization, particle nucleation occurs in the submicron monomer droplets. The fraction of the monomer droplets that is nucleated depends on both the rate of nucleation and on the stability of the monomer droplets against monomer diffusion to growing particles and collision. Any operational variable affecting these processes will have a significant influence on the particle size distribution of the resulting latex, and hence on both the kinetics of the process and the properties of the final product. Thus, Miller et al. [14] reported that in the batch miniemulsion polymerization of styrene, the fraction of miniemulsion droplets that are nucleated could be greatly increased with the addition of a small amount of polymer to the monomer phase. A drawback of miniemulsion polymerization is that the water-insoluble compound remains in the polymer particle after polymerization and may have a deleterious effect on the properties of the polymer.

This paper considers some developments in miniemulsion polymerization aiming at taking advantage of the miniemulsion polymerization mechanisms whilst minimizing the drawbacks of this technique. Comparisons with conventional emulsion polymerization are presented.

1 Grupo de Ingenieria Quimica, Departamen to de Química Aplicada, Facultad de Ciencias Químicas, Universidad del Pais Vasco, España.

${ }^{2}$ Cen tro de Investigación y Desarrollo en Tecnologia de Pinturas (CIDEPINT). Miembro de la Carrera del Investigador de la CIC. 


\section{HIGHLY CONCENTRATED LATEXES}

High solids contents offer numerous advantages for most commercial applications, e.g. low shipping costs and no need to remove water. In practice, the solids content of a latex is limited by its viscosity. For a monodisperse latex, the viscosity approaches infinity as the volume fraction of the polymer particles approaches 0.62 . On the other hand, polydisperse latexes show a lower viscosity, because the small particles fit within the voids of the array of large particles. Polydispersity is associated with long nucleation periods which are typical of miniemulsion polymerization. For the semicontinuous seeded emulsion polymerization of butyl acrylate in which the monomer was added as a miniemulsion, Tang et al. [8] showed that monomer droplet nucleation took place continuously during the feeding period. Masa et al. [9] compared the emulsion and miniemulsion polymerization of styrene, 2-ethylhexyl acrylate and methacrylic acid $(34 / 60 / 6 \mathrm{wt} / \mathrm{wt})$ at $55 \mathrm{wt} \%$ solids content finding that the viscosities of the neutralized final latexes were 4.5 Pa-s for the emulsion and only $0.7 \mathrm{~Pa} \cdot \mathrm{s}$ for the miniemulsion. This suggests that highly concentrated latexes can be obtained through miniemulsion polymerization.

A 65 wt \% solids content latex was obtained by means of the semicontinuous miniemulsion polymerization of methyl methacrylate (MMA), butyl acrylate (BuA) and vinyl acetate (VAc) (monomer molar ratio MMA/BuA/VAc=35/50/15) using a mixed emulsifier system Alipal CO 436 ( $2 \mathrm{wt} \%$ based on monomers) (ammonium salt of sulfated nonylphenoxy (polyethyleneoxy) ethanol (4 ethyleneoxide), Rhône Poulenc) and Brij 98 (2 wt \%) ( $\mathrm{C}_{16} \mathrm{H}_{31}$-O$\left.\left(\mathrm{C}_{2} \mathrm{H}_{4} \mathrm{O}\right){ }_{18}, \mathrm{ICI}\right)$, and hexadecane $(\mathrm{HD})(2 \mathrm{wt} \%)$ as the hydrophobe [10]. Polymerizations were carried out at $80^{\circ} \mathrm{C}$ initiated by potassium persulfate $(1.04 \mathrm{wt} \%)$ by using miniemulsions for both the initial charge and the feed. The partition of the monomer between the initial charge and the feed was the key operational variable. When a high solids content miniemulsion ( $>55 \mathrm{wt} \%$ ) was used as initial charge, coagulum was formed during the batch polymerization of the initial charge, whereas when a low solids content initial charge was employed, the solids content of the feed was excessive and the sonication cell, where the feed was continuously miniemulsified, plugged. A compromise between these situations was achieved using a $55 \mathrm{wt} \%$ solids content initial charge and a $66.1 \mathrm{wt} \%$ solids content feed. Under these conditions a highly concentrated latex (65 wt \%) of low viscosity (0.44 Pa·s) was obtained.

\section{MINIEMULSION POLYMERIZATION IN CSTRS}

Conventional emulsion polymerizations in continuous stirred tank reactors often show oscillations in conversion and number of polymer particles, $\mathrm{N}_{\mathrm{p}}$. The oscillatory behavior is due to the role of the surfactant in particle nucleation. At the beginning of the process, a large number of small polymer particles is formed, their number being controlled by the amount of emulsifier available in the system. These polymer particles grow by polymerization, and hence their surface area increases, depleting the aqueous phase of emulsifier. Therefore, new particles are not formed and, as the reaction mixture is continuously withdrawn from the reactor, $N_{p}$ decreases. After some time, this process counteracts the increase in size of the particles and the amount of free emulsifier increases, allowing nucleation of new particles. The oscillatory behavior is disadvantageous for both control of the production and product properties. This phenomenon is more acute for low emulsifier concentrations and for polymerizing systems in which the volumetric growth rate of the polymer particles is strongly nonlinear with particle diameter (usually systems with high radical desorption rates). Barnett and Schork [4] reported a case in which the miniemulsion polymerization of MMA in a CSTR did not show oscillations whereas 
the conventional emulsion polymerization exhibited decaying oscillatory transients. Surprisingly, the authors reported that the particle size distribution of the latex resulting from the miniemulsion was indistinguishable from the corresponding conventional emulsion latex.

A more stringent test for the miniemulsion process would be to work with a monomer that gives higher radical desorption rates. Vinyl acetate has this characteristic because of its large monomer chain transfer rate and high water-solubility. Both conventional emulsion and miniemulsion polymerizations of VAc (30 wt \% solids content) were carried out in a $0.47 \mathrm{~L}$ jacketed CSTR at $60^{\circ} \mathrm{C}$. The feed flow rates were controlled by means of weight-based flow controllers, and there was no head space in the reactor to ensure that a constant reactor volume was maintained during the experiments. The residence time was $q=20 \mathrm{~min}$. Sodium lauryl sulfate (SLS) was used as emulsifier and potassium persulfate (KPS) as initiator. Hexadecane, HD, (2 wt $\%$ based on monomer) was used in the miniemulsion polymerization. In this case, the feed was continuously miniemulsified in a sonication cell before entering the reactor.

Figures 1 and 2 present the evolution of the monomer conversion, particle size (dp) measured by dynamic light scattering (DLS)-, and number of polymer particles per $\mathrm{cm}^{3}$ of water for both miniemulsion and conventional emulsion polymerizations for two different emulsifier concentrations. It can be seen that at low emulsifier concentrations (Fig. 1) the particle size of the conventional emulsion latex showed severe oscillations, whereas that of the miniemulsion was constant. In addition, the particle size of the miniemulsion was smaller, i. e. larger number of polymer particles. On the other hand, the oscillations in the monomer conversions were less noticeable. At high emulsifier concentration (Fig. 2) neither the particle size of the conventional emulsion latex nor that of the miniemulsion presented oscillations. The monomer conversions were also constant.

The sustained oscillations of particle diameter at the low emulsifier concentration found in the emulsion polymerization resulted from intermittent nucleation. On the other hand, at the high emulsifier concentration the system never became depleted of emulsifier and hence an almost constant nucleation rate was obtained, maintaining the number of polymer particles in the reactor constant. The low sensitivity of the monomer conversion to the particle number oscillations was due to the fact that, because of the large particle size, the system was under Smith-Ewart case III kinetics $(\bar{n}-3)$ [20]. At the high emulsifier concentration, the particle size decreased making the polymerization rate sensitive to the number of polymer particles $(\overline{\mathbf{n}}-0.4)$ and hence monomer conversion increased with $\mathrm{N}_{\mathrm{p}}$. For both emulsifier concentrations, the miniemulsion process does not present oscillations. The reason for this behavior is that, in this process, a stable (as proved by creaming experiments) monomer miniemulsion was continuously fed into the reactor, and hence the total interfacial surface area of the system was quite constant throughout the process preventing oscillations of free emulsifier level. In addition, the monomer droplets are much larger than the particle precursors formed during nucleation in conventional emulsion polymerization, and for these sizes, the nonlinearity of the volumetric growth rate vs particle size is greatly reduced. Comparison between Figures 1 and 2 shows that for both emulsion and miniemulsion polymerizations, $\mathrm{N}_{\mathrm{p}}$ increased with the amount of emulsifier, this effect being more acute for the conventional emulsion polymerization. In the emulsion process, the number of stabilized particle precursors increased when the surfactant level increased. On the other hand, in the case of miniemulsion polymerization, at higher emulsifier concentration the monomer droplet diameter decreased [18], and therefore, a higher number of droplets could be nucleated. Figure 3 presents the molecular weight distribution (MWD) of samples collected at $\mathrm{t} / \mathrm{q}=10$ in the miniemulsion and conventional emulsion polimerizations carried out at low 

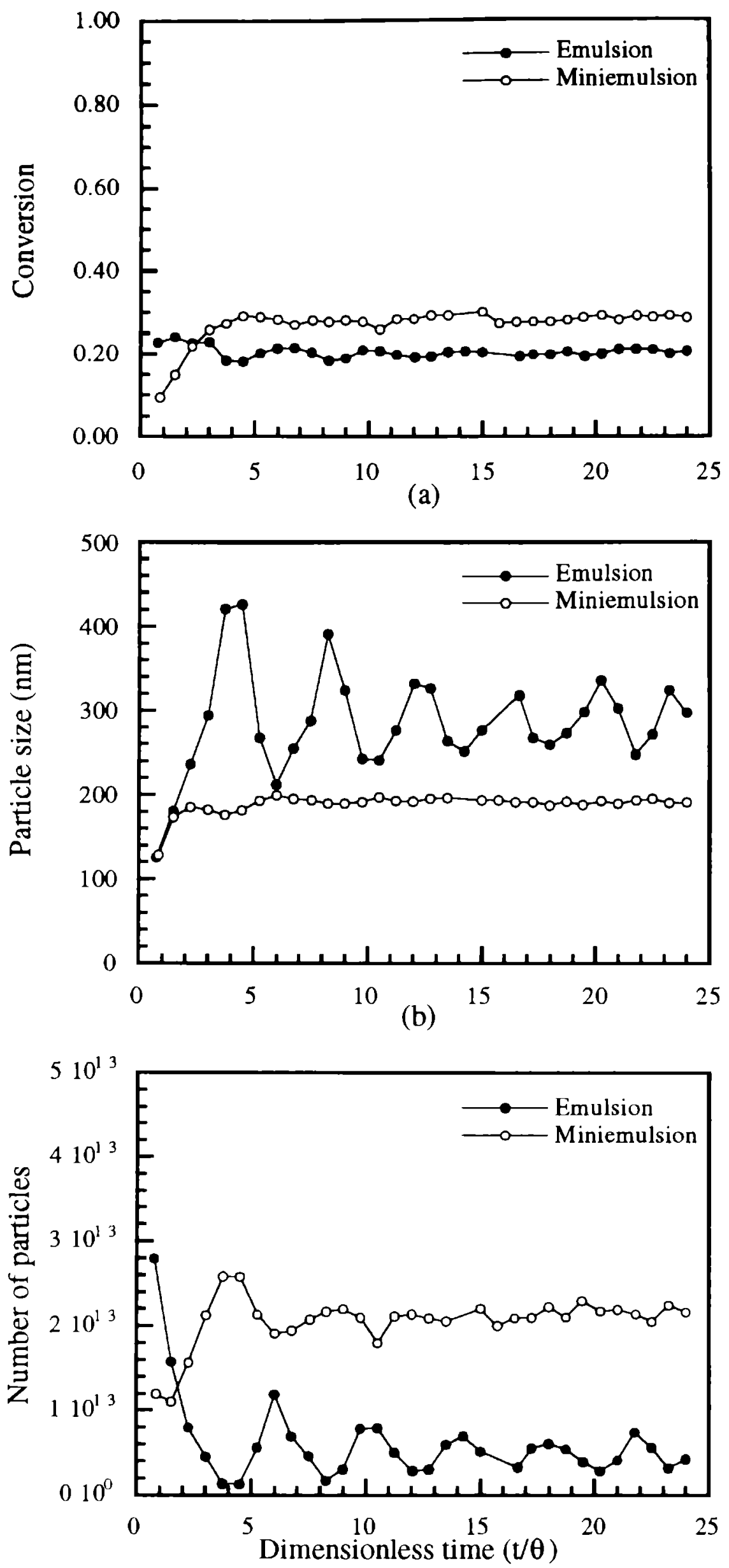

(c)

Fig. 1.- Evolution of conversion (a), particle size (b) and number of polymer particles (c) for the miniemulsion and conventional emulsion polymerization of VAc in a CSTR.

[KPS] $=0.4 \mathrm{wt} \%$; [SLS] = $0.6 \mathrm{wt} \%$ (both based on monomer). 
emulsifier concentration. It can be seen that the MWD was not affected by the process used suggesting that the MWD was controlled by chain transfer to monomer.
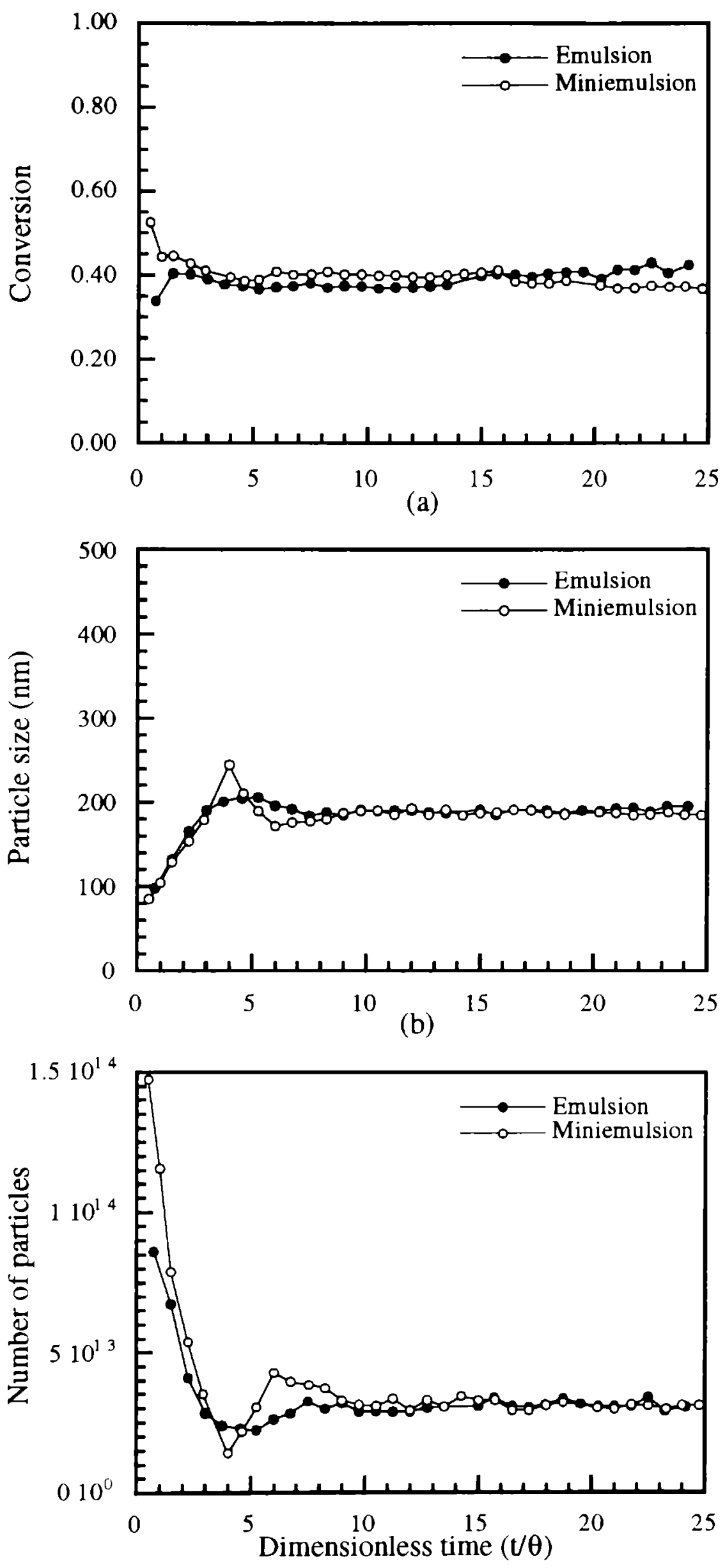

(c)

Fig. 2.- Evolution of conversion (a), particle size (b) and number of polymer particles (c) for the miniemulsion and conventional emulsion polymerization of VAc in a CSTR.

$[\mathrm{KPS}]=0.4 \mathrm{wt} \%$; [SLS] $=4.8 \mathrm{wt} \%$ (both based on monomer). 


\section{ELIMINATION OF THE LOW MOLECULAR WEIGHT HYDROPHOBE}

Fatty alcohols [15-17] and long chain alkane [5] have commonly been used to stabilize the miniemulsion droplets against monomer diffusion. These are low molecular weight compounds that remain in the particles after polymerization plasticizing the polymer, and hence affecting its properties. This effect may be reduced if the hydrophobe becomes covalently bonded to the polymer during polymerization or if a preformed polymer is used as hydrophobe.

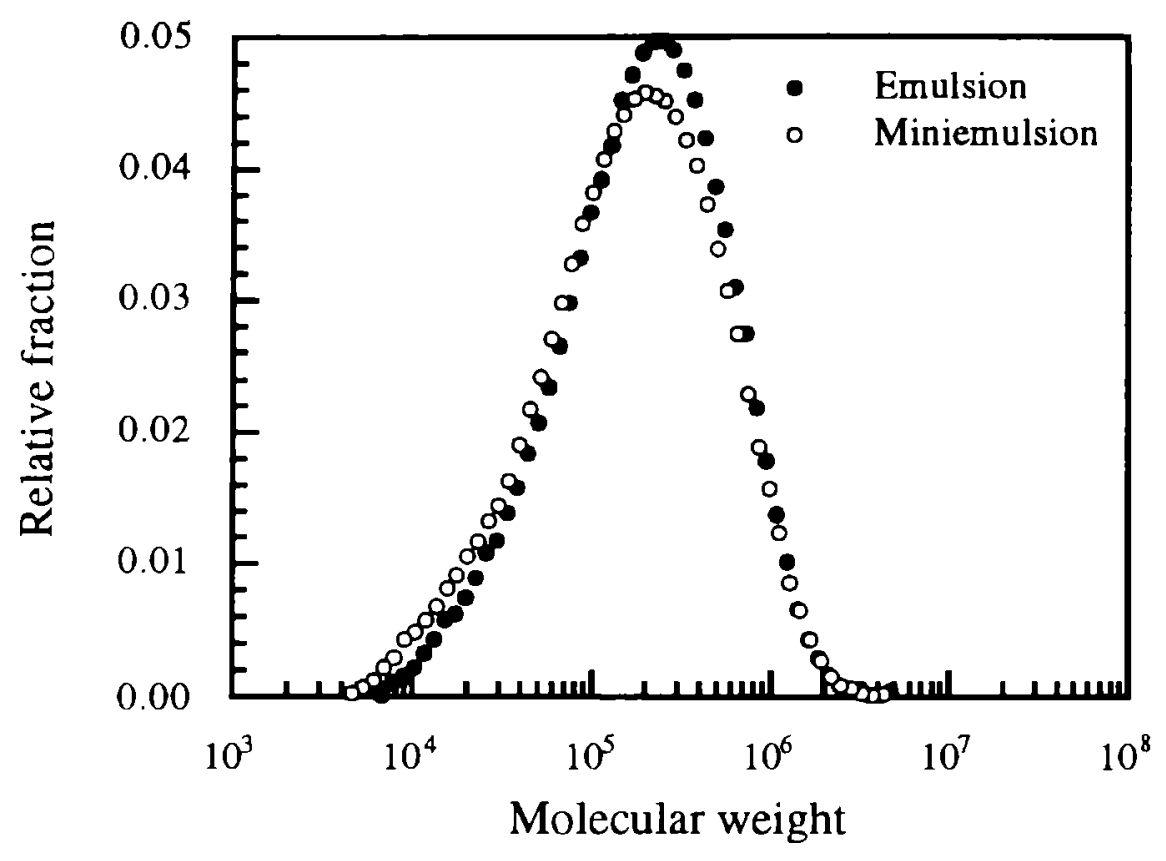

Fig. 3. -Molecular weight distribution for the miniemulsion and conventional emulsion polymerization of VAc in a CSTR $[\mathrm{KPS}]=0.4 \mathrm{wt} \%$; [SLS] $=0.6 \mathrm{wt} \%$ (both based on monomer).

\section{Covalently Bonded Hydrophobe}

This might, in principle, be achieved by using water-insoluble macromonomers, initiators or chain transfer agents. Alduncin et al. [12] studied the ability of a series of initiators with different water solubilities (lauroyl peroxide (LPO), benzoyl peroxide (BPO), and azobis(isobutyronitrile) (AIBN)) in stabilizing monomer droplets against degradation by molecular diffusion in the batch miniemulsion polymerization of styrene. A $2.5 \mathrm{wt} \%$ of initiator based on monomer was used. For the sake of comparison, another series of miniemulsion polymerizations was carried out, in which the stability of the monomer droplets was ensured by using hexadecane in addition to the oil-soluble initiators. Figure 4 presents the particle size distribution (PSD) of the final latexes. It can be seen that, in the absence of HD, only the LPO was sufficiently water-insoluble to avoid extensive monomer droplet degradation, yielding a PSD similar to that of the miniemulsion polymerization costabilized with $\mathrm{HD}$. On the other hand, BPO and AIBN are not water-insoluble enough to avoid the Ostwald ripening effect, and extensive monomer droplet degradation occurred. Figure 4 also shows that the type of initiator had only a limited effect on the PSDs obtained in the miniemulsions costabilized with HD. However, the process yielded a rather low molecular weight polymer $(\overline{\mathrm{Mn}} \sim 40,000)$ [13]. Therefore, the application of this type of process should be restricted to systems in which the MWD is controlled by chain transfer to monomer (for example PVC production). 

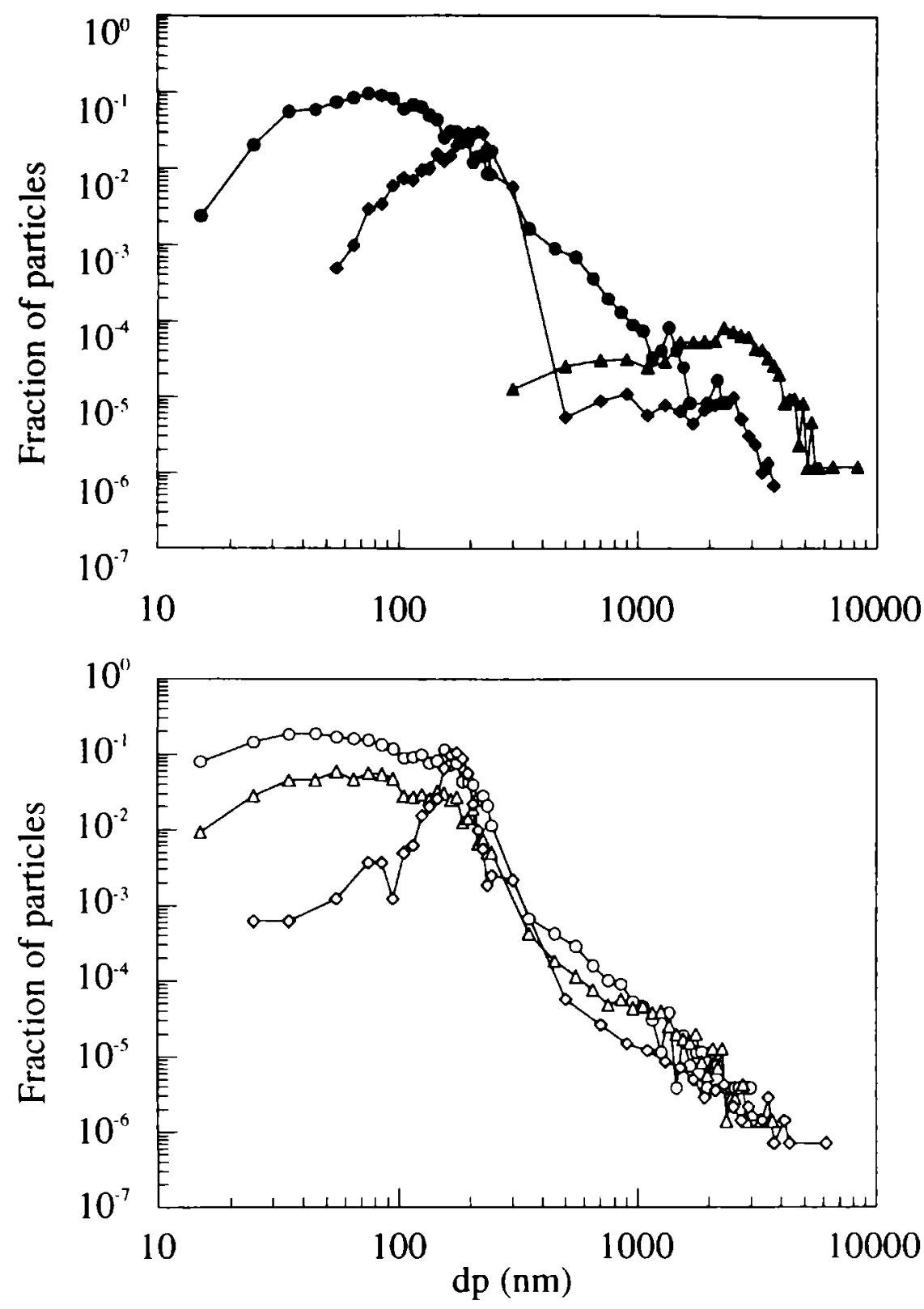

Fig. 4. Particle size distributions of the final latexes obtained in miniemulsion polymerizations initiated by

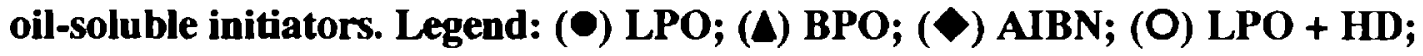
$(\Delta)$ BPO + HD; $(\diamond)$ AIBN + HD.

\section{Polymeric Hydrophobe}

The presence of polymer in the miniemulsion droplets has been reported to increase significantly the polymerization rate by increasing the rate of particle nucleation [14-19]. For the batch miniemulsion polymerization of styrene, Miller et al. [15] found that the larger increase was obtained using a combination of polystyrene and cetyl alcohol although a significant increase was also achieved using only polystyrene. Similar results were obtained by Reimers and Schork [19] in the study of the batch miniemulsion polymerization of methyl methacrylate when poly(methyl methacrylate) was used as hydrophobe. On the other hand, Wang and Schork [18] did not obtain stable vinyl acetate miniemulsions using poly(vinyl acetate) as hydrophobe and polyvinyl alcohol as surfactant. Vinyl acetate miniemulsions ( $55 \mathrm{wt} \%$ solids content stabilized with pVAc, HD and $\mathrm{HD}+\mathrm{pVAc}$ (hydrophobe) and SLS (emulsifier) have been prepared in our laboratories. The miniemulsions containing HD were stable for several days, whereas that using only pVAc as hydrophobe suffered phase separation after some minutes. Nevertheless, these miniemulsions were polymerized in batch, minimizing the time ellapsed between emulsification and polymerization. Conventional emulsion polymerization was carried out as a reference. Coagulum 
free latexes were obtained in all cases with the following particle diameters (measured by DLS): $d_{p}($ emulsion $)=104 \mathrm{~nm} ; d_{p}(H D)=229 \mathrm{~nm} ; d_{p}(H D+p V A c)=219 \mathrm{~nm} ; d_{p}(p V A c)=165 \mathrm{~nm}$.

These results suggest that the pVAc was able to stabilize the miniemulsion droplets long enough for them to be nucleated. In order to test this point, a $55 \mathrm{wt} \%$ solids content miniemulsion (using pVAc as the sole hydrophobe) and a conventional emulsion polymerization were carried out in a $0.47 \mathrm{~L}$ CSTR. Polymerization conditions were as follows: $\mathrm{T}=60{ }^{\circ} \mathrm{C} ; \mathrm{q}=$ $20 \mathrm{~min}$; [Arkopal N 230] = $1 \mathrm{wt} \%$; [Alipal CO436] $=0.75 \mathrm{wt} \%$; [Hydroxyethyl cellulose] $=0.1$ wt \%; [pVAc] $=1 \mathrm{wt} \% ;[\mathrm{KPS}]=1.05 \mathrm{wt} \%$ (all of them based on monomer). The results are presented in Fig. 5. It can be seen that a stable operation was achieved using the miniemulsion process whereas severe oscillations occurred in the conventional emulsion polymerization. This is a proof that nucleation occurred in monomer droplets. It is interesting to notice that $\mathrm{N}_{\mathrm{p}}$ for the miniemulsion process was larger than for the emulsion polymerization, while the opposite was found in a batch reactors. Work is in progress to clarify this point using a complete mathematical model.
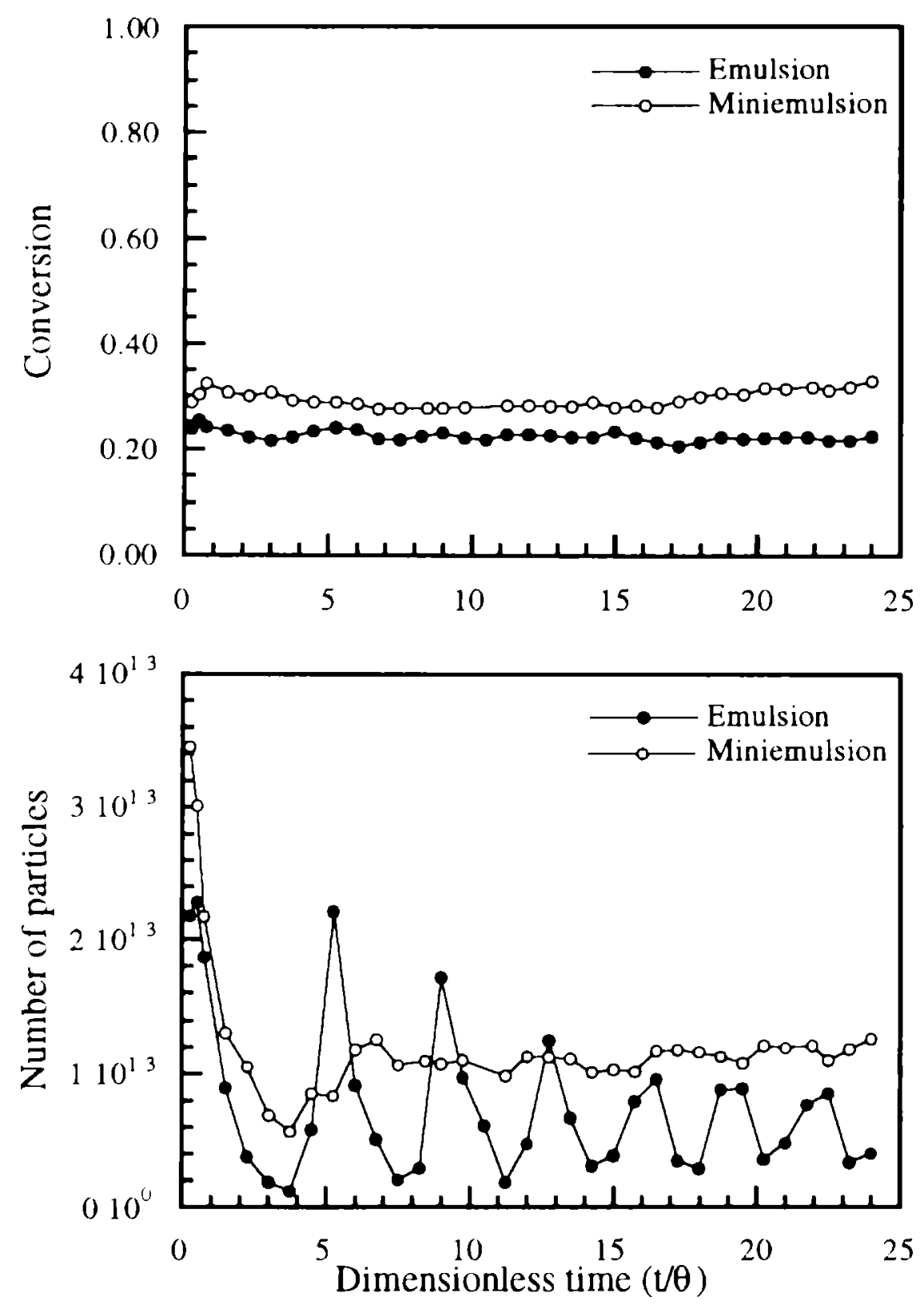

Fig. 5.- Evolution of conversion and number of particles for the miniemulsion and conventional emulsion polymerization of VAc in a CSTR with pVAc as the sole hydrophobe. 


\section{ACKNOWLEDGEMENTS}

Financial support from the Diputacion Foral de Gipuzkoa and Universidad del Pais Vasco (UPV 221.215-TA202/95) and the fellowships for I. Aizpurua by the Basque Government and for J. I Amalvy by AECI-UPV are gratefully appreciated.

\section{REFERENCES}

[1] J. Ugelstad, M. S. El-Aasser, J. W. Vanderhoff.- J. Polym. Sci., Polym. Lett. 11, 503 (1973).

[2] F. K. Hansen, J. Ugelstad.- J. Polym. Sci., Polym. Chem. Ed. 17, 3069 (1979).

[3] Y. T. Choi, M. S. El-Aasser, E. D. Sudol, J. W. Vanderhoff.- J. Polym. Sci., Polym. Chem Ed. 23, 2973 (1985).

[4] D. T. Barnett, F. J. Schork.- Chem. Eng. Comm. 80, 113 (1989).

[5] J. Delgado, M. S. El-Aasser, C. A. Silebi, J. W. Vanderhoff.- J. Polym. Sci., Polym. Chem. Ed. 28, 777 (1990).

[6] J. Delgado, M. S. El-Aasser.- Makromol. Chem. Macromol. Symp. 31, 63 (1990).

[7] J. M. Asua, V. S. Rodriguez, C. A. Silebi, M. S. El-Aasser.- Makromol. Chem., Macromol. Symp. 35/36, 59 (1990).

[8] P.L. Tang, E. D. Sudol, M. E. Adams, M. S. El-Aasser, J. M. Asua.- J. Appl. Polym. Sci. 42, 2019 (1991).

[9] J. A. Masa, L. López de Arbina, J. M. Asua.- J. Appl. Polym. Sci 48, 205 (1993).

[10] M. J. Unzue, J. M. Asua.- J. Appl. Polym Sci. 49, 81 (1993).

[11] K. Fontenot, F. J. Schork.- J. Appl. Polym. Sci. 49, 633 (1993).

[12] J. A. Alduncin, J. Forcada, J. M. Asua.- Macromolecules 27, 2256 (1994).

[13] J. A. Alduncin, J. M. Asua.- Polymer 35, 3758 (1994).

[14] C. M. Miller, P. J. Blythe, E. D. Sudol, C. A. Silebi, M. S. El-Aasser.- J. Polym. Sci.:Part A: Polym. Chem. 32, 2365 (1994).

[15] C. M. Miller, E. D. Sudol, C. A. Silebi, M. S. El-Aasser.- Macromolecules 28, 2754 (1995).

[16] C. M. Miller, E. D. Sudol, C. A. Silebi, M. S. El-Aasser.- Macromolecules 28, 2765 (1995). 
[17] C. M. Miller, E. D. Sudol, C. A. Silebi, M. S. El-Aasser.- Macromolecules 28, 2772 (1995).

[18] S. Wang, F. J. Schork.- J. Appl. Polym. Sci. 54, 2157 (1994).

[19] J. L. Reimers, F. J. Schork. - J. Appl. Polym. Sci. 60, 251 (1996).

[20] W. J. Smith, R. M. Ewart.- J. Chem. Phys. 16, 592 (1948). 